\title{
Reachable Set Bounding for Homogeneous Nonlinear Systems with Delay and Disturbance
}

\author{
Xingao Zhu' ${ }^{1}$ and Yuangong Sun $\mathbb{D}^{1,2,3}$ \\ ${ }^{1}$ School of Mathematical Sciences, University of Jinan, Jinan 250022, China \\ ${ }^{2}$ School of Mathematical Sciences, Qufu Normal University, Qufu 273165, China \\ ${ }^{3}$ College of Mathematics and Systems Science, Shandong University of Science and Technology, Qingdao 266590, China
}

Correspondence should be addressed to Yuangong Sun; sunyuangong@163.com

Received 18 April 2019; Accepted 13 June 2019; Published 1 July 2019

Guest Editor: Raúl Villafuerte-Segura

Copyright (c) 2019 Xingao Zhu and Yuangong Sun. This is an open access article distributed under the Creative Commons Attribution License, which permits unrestricted use, distribution, and reproduction in any medium, provided the original work is properly cited.

\begin{abstract}
Reachable set bounding for homogeneous nonlinear systems with delay and disturbance is studied. By the usage of a new method for stability analysis of positive systems, an explicit necessary and sufficient condition is first derived to guarantee that all the states of positive homogeneous time-delay systems with degree $p>1$ converge asymptotically within a specific ball. Furthermore, the main result is extended to a class of nonlinear time variant systems. A numerical example is given to demonstrate the effectiveness of the obtained results.
\end{abstract}

\section{Introduction}

Recent years have witnessed a rapid development of reachable set bounding for linear systems in [1-11], to name a few. In most of existing references, the traditional LyapunovKrasovskii function method is most commonly used. However, such a method is usually difficult to derive explicit conditions for reachable set estimation of nonlinear systems with delay and disturbance.

Due to the ubiquitous existence of time delay in practical engineering and its adverse effect on stability [12-15] and oscillation [16-19], it has attracted wide attention in recent years. So far, less attention has been paid to reachable set bounding for nonlinear time-delay systems. Such a problem was discussed in $[20,21]$ for certain nonlinear perturbed systems with delay, where the involved nonlinear terms satisfy a linear growth condition. Reachable set bounding for continuous-time and discrete-time homogeneous time-delay positive systems of degree one was studied in [22]. The decay rates of homogeneous positive systems of any degree with time-varying delays were given in [23]. Recently, the same problem was considered in [24] for homogeneous positive systems of degree $p>1$, while time delay was not taken into consideration. The problem of reachable set estimation of switched positive systems with discrete and distributed delays subject to bounded disturbances was investigated in [25].

Positive systems are dynamical systems whose states remain nonnegative whenever the initial states are nonnegative $([26,27])$. In view of the special structure of positive systems, a special method was commonly used for stability analysis of positive systems in [28-33], which is different from the traditional Lyapunov-Krasovskii function method.

Motivated by the work in $[23,24]$, we study in this paper reachable set bounding for homogeneous nonlinear timedelay systems with bounded disturbance. By developing the methods used in [23, 24], we first establish a necessary and sufficient condition such that all the solutions of positive homogeneous time-delay systems with degree $p>1$ converge asymptotically within a specific ball, which contains those results in $[23,24]$ in special cases. The main result is also applied to certain nonlinear time variant systems with delay and disturbance.

Throughout this paper, $\mathbb{R}^{n}$ is the set of $n$-dimensional real vectors. Denote by $x_{i}$ the $i$ th coordinate of $x \in \mathbb{R}^{n}$ for $i \in\langle n\rangle=\{1,2, \ldots, n\}$. Given $x, y \in \mathbb{R}^{n}$, say $x>y$ (or $y<x$ ) if $x_{i}>y_{i}, x \geq y$ (or $y \preceq x$ ) if $x_{i} \geq y_{i}, i \in\langle n\rangle$. Denote $\mathbb{R}_{+}^{n}=\left\{x \in \mathbb{R}^{n}: x \geq 0\right\}$. For $x=\left(x_{i}\right) \in \mathbb{R}^{n}$, 
denote $|x|=\left(\left|x_{i}\right|\right) \in \mathbb{R}_{+}^{n}$ and $\|x\|_{\infty}=\max _{i \in\langle n\rangle}\left|x_{i}\right|$. Let $\mathscr{B}(\varepsilon)=\left\{x \in \mathbb{R}^{n} \mid\|x\|_{\infty} \leq \varepsilon\right\}$, where $\varepsilon>0$ is a constant. For given $r>0$, denote $\mathbb{B F}_{r}\left([0, \infty], \mathbb{R}^{n}\right)=\{\omega:[0, \infty] \longrightarrow$ $\left.\mathbb{R}^{n} \mid\|\omega(t)\|_{\infty} \leq r, \forall t \geq 0\right\}$. An $n \times n$-dimensional matrix $A$ is called Metzler if all its off-diagonal entries are nonnegative.

\section{Preliminaries}

In this paper, nonlinear time-delay systems of the form

$$
\begin{aligned}
& \dot{x}(t)=f(x(t))+g((x(t-\tau(t)))+\omega(t), \quad t \geq 0 \\
& x(t)=\varphi(t), \quad t \in[-h, 0],
\end{aligned}
$$

are investigated, where $x(t) \in \mathbb{R}^{n}$ is the state vector, $f, g$ : $\mathbb{R}^{n} \longrightarrow \mathbb{R}^{n}$ are continuous vector functions satisfying $f(0)=$ $g(0)=0, \tau(t)$ is a time delay satisfying $0 \leq \tau(t) \leq h, h>0$ is a constant, $\omega(t) \in \mathbb{B F}_{r}\left([0, \infty], \mathbb{R}^{n}\right)$ is the disturbance, and the initial state $\varphi(t):[-h, 0] \longrightarrow \mathbb{R}^{n}$ is continuous. Note that when $\tau(t) \equiv 0$, system (1) takes the form of the system considered in [24].

The following definitions and lemma in [34] will be required.

Definition 1. Assume that $f: \mathbb{R}^{n} \longrightarrow \mathbb{R}^{n}$ is continuous on $\mathbb{R}^{n}$ and continuously differentiable on $\mathbb{R}^{n} \backslash\{0\}$. The vector function $f$ is called cooperative if the Jacobian matrix $(\partial f / \partial x)(x), x \in \mathbb{R}^{n} \backslash\{0\}$, is Metzler.

Definition 2. A vector function $f: \mathbb{R}^{n} \longrightarrow \mathbb{R}^{n}$ is called homogeneous of degree $p>0$ if $f(\lambda x)=\lambda^{p} f(x), x \in \mathbb{R}^{n}$, $\lambda>0$.

Definition 3. A vector function $g: \mathbb{R}^{n} \longrightarrow \mathbb{R}^{n}$ is called orderpreserving on $\mathbb{R}_{+}^{n}$ provided that $g(x) \geq g(y)$, where $x, y \in$ $\mathbb{R}_{+}^{n}, x \geq y$.

Lemma 4. A cooperative vector function $f$ satisfies $f_{i}(u) \geq$ $f_{i}(v)$, where $u, v \in \mathbb{R}^{n} \backslash\{0\}, u \geq v, u_{i}=v_{i}, i \in\langle n\rangle$.

In this paper, we need the following assumptions:

(H1) $f$ and $g$ are continuously differentiable on $\mathbb{R}^{n} \backslash\{0\}$ and homogeneous of degree $p>1$;

(H2) $f$ is cooperative and $g$ is order-preserving on $\mathbb{R}_{+}^{n}$;

(H3) $\omega(t) \succeq 0$ for $t \geq 0$.

Following the proof given in [22], we can easily obtain the following lemma.
Lemma 5. System (1) is positive under assumptions (H2) and (H3).

\section{Main Results}

Theorem 6. Suppose that (H1)-(H3) are valid. Then, we have the following equivalent statements:

(i) There is an $n$-dimensional vector $v>0$ satisfying $f(v)+$ $g(v) \prec 0$.

(ii) The solution $x(t)$ of system (1) satisfies

$$
\|x(t)\|_{\infty} \leq \alpha+(\beta+\gamma t)^{-1 /(p-1)}
$$

for any $t \geq 0$, any initial state $\varphi(t) \in \mathscr{C}\left([-h, 0], \mathbb{R}_{+}^{n}\right)$, any disturbance $\omega(t) \in \mathbb{B F}_{r}\left([0, \infty], \mathbb{R}_{+}^{n}\right)$, and any bounded delay $\tau(t)$, where $\alpha, \beta$, and $\gamma$ are appropriate nonnegative constants dependent on $r, h$, and the initial state $\varphi$, and $\alpha=0$ if $r=0$.

In addition, if condition ( $i$ ) holds, $\alpha, \beta$, and $\gamma$ can be chosen as follows:

$$
\begin{aligned}
& \alpha=\theta \rho, \\
& \beta=(K \rho)^{1-p}, \\
& \gamma=(p-1) \eta \rho^{1-p},
\end{aligned}
$$

where $\rho=\max _{i \in\langle n\rangle} v_{i}$,

$$
\begin{gathered}
\theta=\left(\frac{r}{-\max _{i \in\langle n\rangle}\left[f_{i}(v)+g_{i}(v)\right]}\right)^{1 / p}, \\
K= \begin{cases}0, & \|\varphi\|_{v} \leq \theta, \\
{\left[\left(\|\varphi\|_{v}\right)^{p}-\theta^{p}\right]^{1 / p},} & \|\varphi\|_{v}>\theta,\end{cases}
\end{gathered}
$$

$\|\varphi\|_{v}=\max _{i \in\langle n\rangle, t \in[-h, 0]}\left(\left|\varphi_{i}(t)\right| / v_{i}\right), \eta$ satisfies $0<\eta<$ $\min _{i \in\langle n\rangle} \eta_{i}$, and $\eta_{i}$ satisfies the following equation:

$$
\begin{aligned}
\frac{f_{i}(v)}{v_{i}}+\frac{g_{i}(v)}{v_{i}}\left[1+(p-1) K^{p-1} \eta_{i} h\right]^{p /(p-1)}+\eta_{i} & =0, \\
i & \in\langle n\rangle .
\end{aligned}
$$

Proof. (i) $\Longrightarrow$ (ii) Given the initial state $\varphi \in \mathscr{C}\left([-h, 0], \mathbb{R}_{+}^{n}\right)$, from Lemma 5 we have $x(t) \geq 0, t \geq 0$. Based on definitions of $K$ and $\|\varphi\|_{v}$, we have

$$
\frac{x_{i}(t)}{v_{i}} \leq\left(\theta^{p}+K^{p}\right)^{1 / p}, \quad t \in[-h, 0], i \in\langle n\rangle .
$$

Set

$$
z_{i}(t)= \begin{cases}\frac{x_{i}(t)}{v_{i}}-\left\{\theta^{p}+\left[K^{1-p}+(p-1) \eta t\right]^{-p /(p-1)}\right\}^{1 / p}, & t \geq 0, i \in\langle n\rangle, \\ \frac{x_{i}(t)}{v_{i}}-\left(\theta^{p}+K^{p}\right)^{1 / p}, & t \in[-h, 0], i \in\langle n\rangle .\end{cases}
$$


Then (6) and (7) yield $z_{i}(t) \leq 0, t \in[-h, 0], i \in\langle n\rangle$. Next, we show that $z_{i}(t) \leq 0$ for $i \in\langle n\rangle$ and $t \geq 0$. If it is not true, there is a constant $t_{*} \geq 0$ and an index $k \in\langle n\rangle$ guaranteeing $z_{i}(t) \leq 0$ for $i \in\langle n\rangle, t \in\left[0, t_{*}\right]$, and $z_{k}\left(t_{*}\right)=0$. Therefore,

$$
\begin{aligned}
& \dot{z}_{k}\left(t_{*}\right) \geq 0, \\
& \frac{x_{i}(t)}{v_{i}} \leq\left\{\theta^{p}+\left[K^{1-p}+(p-1) \eta t\right]^{-p /(p-1)}\right\}^{1 / p}, \\
& t \in\left[0, t_{*}\right], \quad i \in\langle n\rangle . \\
& \frac{x_{k}\left(t_{*}\right)}{v_{k}}=\left\{\theta^{p}+\left[K^{1-p}+(p-1) \eta t_{*}\right]^{-p /(p-1)}\right\}^{1 / p} .
\end{aligned}
$$

Using Lemma 4 and the homogeneity of $f$, we get from (9) and (10) that

$$
\begin{aligned}
f_{k} & \left(x\left(t_{*}\right)\right) \\
& \leq f_{k}\left(\left\{\theta^{p}+\left[K^{1-p}+(p-1) \eta t_{*}\right]^{-p /(p-1)}\right\}^{1 / p} v\right) \\
& =\left\{\theta^{p}+\left[K^{1-p}+(p-1) \eta t_{*}\right]^{-p /(p-1)}\right\} f_{k}(v) \\
& =\theta^{p} f_{k}(v)+\left[K^{1-p}+(p-1) \eta t_{*}\right]^{-p /(p-1)} f_{k}(v) .
\end{aligned}
$$

For the case when $\tau\left(t_{*}\right) \leq t_{*}$, it holds that

$$
\begin{aligned}
& \frac{x_{i}\left(t_{*}-\tau\left(t_{*}\right)\right)}{v_{i}} \\
& \leq\left\{\theta^{p}+\left[K^{1-p}+(p-1) \eta\left(t_{*}-\tau\left(t_{*}\right)\right)\right]^{-p /(p-1)}\right\}^{1 / p}, \\
& i \in\langle n\rangle .
\end{aligned}
$$

Considering $g$ is homogeneous and order-preserving, we conclude

$$
\begin{aligned}
g_{k} & \left(x\left(t_{*}-\tau\left(t_{*}\right)\right)\right) \leq g_{k}\left(\left\{\theta^{p}\right.\right. \\
+ & {\left.\left.\left[K^{1-p}+(p-1) \eta\left(t_{*}-\tau\left(t_{*}\right)\right)\right]^{-p /(p-1)}\right\}^{1 / p} v\right) } \\
= & \left\{\theta^{p}+\left[K^{1-p}+(p-1) \eta\left(t_{*}-\tau\left(t_{*}\right)\right)\right]^{-p /(p-1)}\right\} \\
& \cdot g_{k}(v)=\theta^{p} g_{k}(v)+\left[K^{1-p}+(p-1) \eta\left(t_{*}\right.\right. \\
& \left.\left.-\tau\left(t_{*}\right)\right)\right]^{-p /(p-1)} g_{k}(v) .
\end{aligned}
$$

Note that

$$
\begin{aligned}
& {\left[K^{1-p}+(p-1) \eta\left(t_{*}-\tau\left(t_{*}\right)\right)\right]^{-p /(p-1)}} \\
& =\left[K^{1-p}+(p-1) \eta t_{*}\right]^{-p /(p-1)} \\
& \quad \times\left[\frac{\eta(p-1) \tau\left(t_{*}\right)}{K^{1-p}+\eta(p-1)\left(t_{*}-\tau\left(t_{*}\right)\right)}+1\right]^{p /(p-1)} \\
& \leq\left[K^{1-p}+(p-1) \eta t_{*}\right]^{-p /(p-1)} \\
& \quad \times\left[1+(p-1) K^{p-1} \eta h\right]^{p /(p-1)} .
\end{aligned}
$$

We further get from (13) and (14) that

$$
\begin{aligned}
g_{k} & \left(x\left(t_{*}-\tau\left(t_{*}\right)\right)\right) \leq \theta^{p} g_{k}(v) \\
+ & {\left[K^{1-p}+\eta(p-1) t_{*}\right]^{-p /(p-1)} } \\
\cdot & {\left[1+(p-1) K^{p-1} \eta h\right]^{p /(p-1)} g_{k}(v) . }
\end{aligned}
$$

For the case when $\tau\left(t_{*}\right)>t_{*}$, it holds that $z_{i}\left(t_{*}-\tau\left(t_{*}\right)\right) \leq 0$; i.e.,

$$
\frac{x_{i}\left(t_{*}-\tau\left(t_{*}\right)\right)}{v_{i}} \leq\left(\theta^{p}+K^{p}\right)^{1 / p}, \quad i \in\langle n\rangle .
$$

It thus follows that

$$
\begin{aligned}
g_{k} & \left(x\left(t_{*}-\tau\left(t_{*}\right)\right)\right) \leq\left(\theta^{p}+K^{p}\right) g_{k}(v)=\theta^{p} g_{k}(v) \\
+ & {\left[K^{1-p}+(p-1) \eta t_{*}\right]^{-p /(p-1)} } \\
\cdot & K^{p}\left[K^{1-p}+(p-1) \eta t_{*}\right]^{p /(p-1)} g_{k}(v) \leq \theta^{p} g_{k}(v) \\
+ & {\left[K^{1-p}+(p-1) \eta t_{*}\right]^{-p /(p-1)} } \\
\cdot & {\left[1+(p-1) K^{p-1} \eta h\right]^{p /(p-1)} g_{k}(v) . }
\end{aligned}
$$

Next, we can conclude from (1) and (7) that

$$
\begin{aligned}
& \dot{z}_{k}\left(t_{*}\right)=\frac{f_{k}\left(x\left(t_{*}\right)\right)+g_{k}\left(x\left(t_{*}-\tau\left(t_{*}\right)\right)\right)+w_{k}\left(t_{*}\right)}{v_{k}} \\
& +\eta\left\{\theta^{p}+\left[K^{1-p}+(p-1) \eta t_{*}\right]^{-p /(p-1)}\right\}{ }^{(1-p) / p} \\
& \cdot\left[K^{1-p}+(p-1) \eta t_{*}\right]^{(1-2 p) /(p-1)} \\
& \leq \frac{f_{k}\left(x\left(t_{*}\right)\right)+g_{k}\left(x\left(t_{*}-\tau\left(t_{*}\right)\right)\right)+w_{k}\left(t_{*}\right)}{v_{k}} \\
& +\eta\left[K^{1-p}+(p-1) \eta t_{*}\right]^{-p /(p-1)} .
\end{aligned}
$$


Consequently, (11), (15), (17), and (18) imply that

$$
\begin{aligned}
& \dot{z}_{k}\left(t_{*}\right) \leq \frac{\theta^{p}\left[f_{k}(v)+g_{k}(v)\right]+r}{v_{k}}+\left[K^{1-p}\right. \\
& \left.+(p-1) \eta t_{*}\right]^{-p /(p-1)} \times\left\{\frac{f_{k}(v)}{v_{k}}\right. \\
& \left.+\frac{g_{k}(v)}{v_{k}}\left[1+(p-1) K^{p-1} \eta h\right]^{p /(p-1)}+\eta\right\} .
\end{aligned}
$$

On the other hand, the definitions of $\theta$ and $\eta$ yield that

$$
\begin{aligned}
\theta^{p} & {\left[f_{k}(v)+g_{k}(v)\right]+r \leq \theta^{p} \max _{i \in\langle n\rangle}\left[f_{i}(v)+g_{i}(v)\right]+r } \\
& =0
\end{aligned}
$$

and

$$
\frac{f_{k}(v)}{v_{k}}+\frac{g_{k}(v)}{v_{k}}\left[1+(p-1) K^{p-1} \eta h\right]^{p /(p-1)}+\eta<0 .
$$

Combining this with (19), we have $\dot{z}_{k}\left(t_{*}\right)<0$, which contradicts (8). Therefore, $z_{i}(t) \leq 0, t \geq 0, i \in\langle n\rangle$; i.e.,

$$
\begin{array}{r}
\frac{x_{i}(t)}{v_{i}} \leq\left\{\theta^{p}+\left[K^{1-p}+(p-1) \eta t\right]^{-p /(p-1)}\right\}^{1 / p}, \\
t \geq 0, i \in\langle n\rangle .
\end{array}
$$

From the well-known inequality $(a+b)^{q} \leq a^{q}+b^{q}$ for $a, b \geq 0$ and $0<q<1$, we further get

$$
\begin{aligned}
\frac{x_{i}(t)}{v_{i}} \leq \theta+\left[K^{1-p}+(p-1) \eta t\right]^{-1 /(p-1)} & \\
& t \geq 0, i \in\langle n\rangle .
\end{aligned}
$$

It implies (2).

(ii) $\Longrightarrow$ (i) For the particular case when $r=0$ and $h=0$, system (1) reduces to

$$
\dot{x}(t)=f(x(t))+g(x(t)), \quad t \geq 0 .
$$

Given the initial condition $x(0) \geq 0$, each solution of system (24) satisfies

$$
\|x(t)\|_{\infty} \leq(\beta+\gamma t)^{-1 /(p-1)} .
$$

That is, system (24) is asymptotically stable. Based on Proposition 4.1 in [35], there is a vector $v>0$ such that $f(v)+g(v) \prec$ 0 . The proof is complete.
Remark 7. It can be seen from Theorem 6 that the bound of the reachable set is determined by the bound of disturbances, the choice of $v$, and the value of $p$. When the bound of disturbances and the value of $p$ are given, an appropriate vector $v$ can be chosen to guarantee a minimal bound of the reachable set by solving the following nonlinear optimization problem: $\min _{v>0} \theta$ subject to $f(v)+g(v) \prec 0$, where $\theta$ is defined as in Theorem 6 .

Remark 8. If $\omega(t)=0$ for $t \geq 0$, then Theorem 6 reduces to the main result given in [23]. If $g(x)=0$ for $x \in \mathbb{R}^{n}$, then Theorem 6 reduces to the main result given in [24]. system

Finally, consider the following nonlinear time-varying

$$
\dot{x}(t)=\tilde{f}(t, x(t))+\widetilde{g}(t,(x(t-\tau(t)))+\omega(t),
$$

$t \geq 0$

$$
x(t)=\varphi(t), \quad t \in[-h, 0]
$$

where $x(t), \tau(t), \omega(t)$, and $\varphi(t)$ are the same as in (1), and $\tilde{f}, \tilde{g}$ : $[0, \infty) \times \mathbb{R}^{n} \longrightarrow \mathbb{R}^{n}$ are vector functions satisfying $\tilde{f}(t, 0)=$ $\tilde{g}(t, 0)=0$.

Suppose that $\tilde{f}$ and $\tilde{g}$ satisfy the following assumption:

(H4) $\tilde{f}$ and $\tilde{g}$ are continuous on $[0, \infty) \times \mathbb{R}^{n}$, continuously differentiable with respect to $x$ on $\mathbb{R}^{n} \backslash\{0\}$, and there are vector functions $f$ and $g$ satisfying (H1) and (H2), and for $x_{i} \neq 0$,

$$
\begin{array}{r}
\tilde{f}_{i}(t, x) \operatorname{sign} x_{i} \leq f_{i}(|x|), \\
\left|\tilde{g}_{i}(t, x)\right| \leq g_{i}(|x|),
\end{array}
$$

$$
t \geq 0, i \in\langle n\rangle \text {. }
$$

Without the restriction on the disturbance that $\omega(t) \geq 0$ for $t \geq 0$, we can get the following reachable set bounding criterion for system (26).

Theorem 9. Suppose that (H4) is valid. If there is an $n$ dimensional vector $v>0$ such that $f(v)+g(v) \prec 0$, the solution of system (26) satisfies (2), where constants $\alpha, \beta$, and $\gamma$ are defined by (3).

Proof. Set

$$
y_{i}(t)= \begin{cases}\frac{\left|x_{i}(t)\right|}{v_{i}}-\left\{\theta^{p}+\left[K^{1-p}+(p-1) \eta t\right]^{-p /(p-1)}\right\}^{1 / p}, & t \geq 0, i \in\langle n\rangle, \\ \frac{\left|x_{i}(t)\right|}{v_{i}}-\left(\theta^{p}+K^{p}\right)^{1 / p}, & t \in[-h, 0], i \in\langle n\rangle .\end{cases}
$$




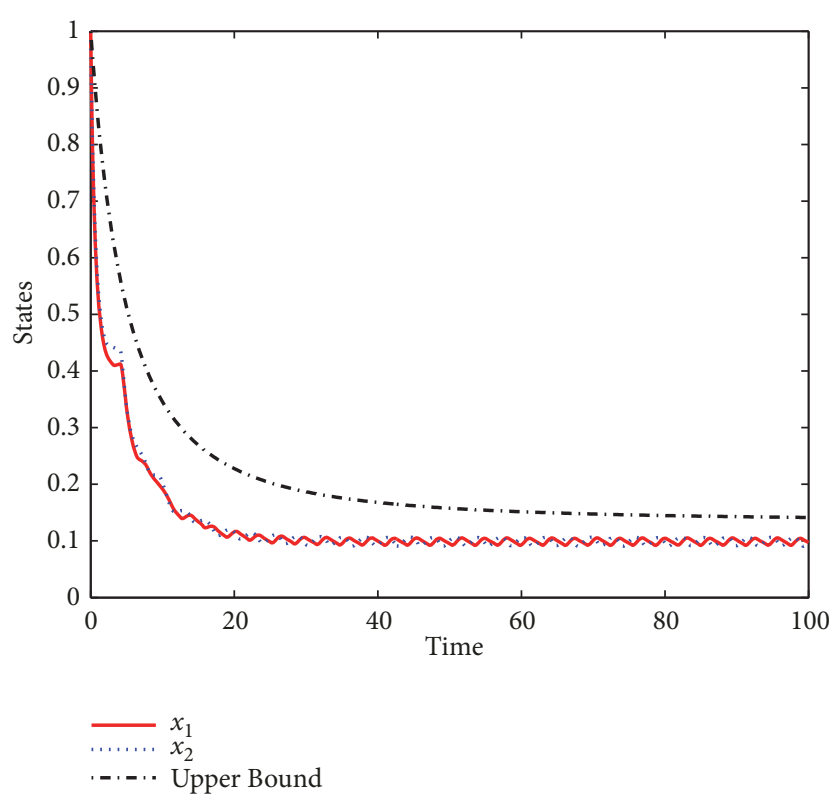

FIgURE 1: The states of system (1).

Based on definitions of $K$ and $\|\varphi\|_{v}$, it holds that $y_{i}(t) \leq 0$, $t \in[-h, 0], i \in\langle n\rangle$. For the case when $x_{i}(t) \neq 0, t \geq 0$, notice that

$$
\begin{aligned}
\dot{y}_{i}(t) & =D_{-}\left|x_{i}(t)\right|=\dot{x}_{i}(t) \operatorname{sign} x_{i}(t) \\
\leq & f_{i}(|x(t)|)+g_{i}(|x(t-\tau(t))|)+\left|w_{i}(t)\right|, \\
& \quad i \in\langle n\rangle .
\end{aligned}
$$

Here $D_{-}$denotes the left derivative. Similar to the analysis in Theorem 6, it is not difficult to conclude that $y_{i}(t) \leq 0, t \geq 0$, $i \in\langle n\rangle$. Consequently, (2) holds. The proof is complete.

\section{Numerical Example}

Consider system (1) with

$$
\begin{aligned}
f\left(x_{1}, x_{2}\right) & =\left(\begin{array}{cc}
-3 & 6 \\
2 & -2
\end{array}\right)\left(\begin{array}{l}
x_{1}^{3 / 2} \\
x_{2}^{3 / 2}
\end{array}\right)-\sqrt{x_{1}^{3}+x_{2}^{3}}\left(\begin{array}{l}
3 \\
1
\end{array}\right), \\
g\left(x_{1}, x_{2}\right) & =\left(\begin{array}{c}
0.2 x_{2}^{3 / 2} \\
0.4 x_{1}^{3 / 2}
\end{array}\right), \\
\omega(t) & =\left(\begin{array}{l}
0.05|\sin t| \\
0.04|\cos t|
\end{array}\right), \\
\tau(t) & =5+\sin t, \quad t \geq 0 .
\end{aligned}
$$

It is easy to verify that assumptions (H1)-(H2) hold. Let $v=$ $(1,1)^{T}$. Then $f(v)+g(v)<0$. By a direct calculation, it yields $h=6, r=0.05, \alpha \approx 0.1345$, and $\gamma \approx 0.11$.

We conclude from Theorem 6 that there is a ball $\mathscr{B}(0.1345)$ such that all the states of system (1) converge asymptotically within it. Given the initial state $\varphi(t)=(1,1)^{T}$, $t \in[-6,0]$, noting that $\|\varphi\|_{v}=1$ and $\beta \approx 1.0749$, solution (1) satisfies

$$
\|x(t)\|_{\infty} \leq 0.1345+(1.0749+0.11 t)^{-2}, \quad t \geq 0 .
$$

Figure 1 presents the simulation.

\section{Conclusion}

This paper has been concerned with reachable set bounding for homogeneous nonlinear time-delay systems with disturbance. We not only derive explicit reachable set bounding criterion independent of delay, but also estimate the decay rate. It will be interesting to extend our work to the case of unbounded delays and discrete-time systems.

\section{Data Availability}

The data used to support the findings of this study are included within the article.

\section{Conflicts of Interest}

We declare that there are no conflicts of interest regarding this paper.

\section{Acknowledgments}

This work is supported by the National Natural Science Foundation of China under grant 61873110.

\section{References}

[1] E. Fridman and U. Shaked, "On reachable sets for linear systems with delay and bounded peak inputs," Automatica, vol. 39, no. 11, pp. 2005-2010, 2003.

[2] J.-H. Kim, "Improved ellipsoidal bound of reachable sets for time-delayed linear systems with disturbances," Automatica, vol. 44, no. 11, pp. 2940-2943, 2008.

[3] Z. Q. Zuo, D. W. C. Ho, and Y. J. Wang, "Reachable set bounding for delayed systems with polytopic uncertainties: the maximal Lyapunov-Krasovskii functional approach," Automatica, vol. 46, no. 5, pp. 949-952, 2010.

[4] O. M. Kwon, S. M. Lee, and J. H. Park, "On the reachable set bounding of uncertain dynamic systems with time-varying delays and disturbances," Information Sciences, vol. 181, no. 17, pp. 3735-3748, 2011.

[5] P. T. Nam and P. N. Pathirana, "Further result on reachable set bounding for linear uncertain polytopic systems with interval time-varying delays," Automatica, vol. 47, no. 8, pp. 1838-1841, 2011.

[6] P. T. Nam, P. N. Pathirana, and H. Trinh, "Exponential convergence of time-delay systems in the presence of bounded disturbances," Journal of Optimization Theory and Applications, vol. 157, no. 3, pp. 843-852, 2013.

[7] N. D. That, P. T. Nam, and Q. P. Ha, "Reachable set bounding for linear discrete-time systems with delays and bounded disturbances," Journal of Optimization Theory and Applications, vol. 157, no. 1, pp. 96-107, 2013.

[8] Z. Zuo, Y. Chen, Y. Wang, D. W. Ho, M. Z. Chen, and H. Li, "A note on reachable set bounding for delayed systems with polytopic uncertainties," Journal of The Franklin Institute, vol. 350, no. 7, pp. 1827-1835, 2013.

[9] J. Lam, B. Zhang, Y. Chen, and S. Xu, "Reachable set estimation for discrete-time linear systems with time delays," International Journal of Robust and Nonlinear Control, 2013. 
[10] B. Zhang, J. Lam, and S. Xu, "Reachable set estimation and controller design for distributed delay systems with bounded disturbances," Journal of The Franklin Institute, vol. 351, no. 6, pp. 3068-3088, 2014.

[11] L. V. Hien and H. M. Trinh, "A new approach to state bounding for linear time-varying systems with delay and bounded disturbances," Automatica, vol. 50, no. 6, pp. 1735-1738, 2014.

[12] J. Qi and Y. Sun, "Global exponential stability of certain switched systems with time-varying delays," Applied Mathematics Letters, vol. 26, no. 7, pp. 760-765, 2013.

[13] G. Zong, H. Ren, and L. Hou, "Finite-time stability of interconnected impulsive switched systems," IET Control Theory \& Applications, vol. 10, no. 6, pp. 648-654, 2016.

[14] Y. Li, Y. Sun, and F. Meng, "New criteria for exponential stability of switched time-varying systems with delays and nonlinear disturbances," Nonlinear Analysis: Hybrid Systems, vol. 26, pp. 284-291, 2017.

[15] Y. Li, Y. Sun, F. Meng, and Y. Tian, "Exponential stabilization of switched time-varying systems with delays and disturbances," Applied Mathematics and Computation, vol. 324, pp. 131-140, 2018.

[16] H. Liu, F. Meng, and P. Liu, "Oscillation and asymptotic analysis on a new generalized Emden-Fowler equation," Applied Mathematics and Computation, vol. 219, no. 5, pp. 2739-2748, 2012.

[17] J. Shao, Z. Zheng, and F. Meng, "Oscillation criteria for fractional differential equations with mixed nonlinearities," Advances in Difference Equations, p. 323, 2013.

[18] J. Shao, F. Meng, and Z. Zheng, "Oscillation theorems for linear matrix Hamiltonian systems," Applied Mathematics and Computation, vol. 253, pp. 402-409, 2015.

[19] H. Liu and F. Meng, "Interval oscillation criteria for secondorder nonlinear forced differential equations involving variable exponent," Advances in Difference Equations, Paper No. 291, 14 pages, 2016.

[20] P. T. Nam, P. N. Pathirana, and H. Trinh, "Reachable set bounding for nonlinear perturbed time-delay systems: the smallest bound," Applied Mathematics Letters, vol. 43, pp. 6871, 2015.

[21] X. Zhu, Y. Sun, and X.-J. Xie, "State bounding for nonlinear time-varying systems with delay and disturbance," Journal of The Franklin Institute, vol. 355, no. 16, pp. 8213-8224, 2018.

[22] N. Zhang, Y. Sun, and P. Zhao, "State bounding for homogeneous positive systems of degree one with time-varying delay and exogenous input," Journal of The Franklin Institute, vol. 354, no. 7, pp. 2893-2904, 2017.

[23] J. G. Dong, "On the decay rates of homogeneous positive systems of any degree with time-varying delays," Institute of Electrical and Electronics Engineers Transactions on Automatic Control, vol. 60, no. 11, pp. 2983-2988, 2015.

[24] Yuangong Sun and Fanwei Meng, "Reachable Set Estimation for a Class of Nonlinear Time-Varying Systems," Complexity, vol. 2017, Article ID 5876371, 6 pages, 2017.

[25] D. Tian, S. Liu, Y. Sun, and J. Xia, "Reachable set estimation for switched positive systems with mixed time-varying delays and bounded disturbances," IET Control Theory \& Applications, vol. 12, no. 15, pp. 2003-2009, 2018.

[26] L. Farina and S. Rinaldi, Positive Linear Systems: Theory and Applications, Wiley, New York, NY, USA, 2000.

[27] T. Kaczorek, Positive $1 D$ and 2D Systems, Springer-Verlag, New York, NY, USA, 2002.
[28] X. Liu, W. Yu, and L. Wang, "Stability analysis of positive systems with bounded time-varying delays," IEEE Transactions on Circuits and Systems II: Express Briefs, vol. 56, no. 7, pp. 600604, 2009.

[29] X. Liu, W. Yu, and L. Wang, "Stability analysis for continuoustime positive systems with time-varying delays," IEEE Transactions on Automatic Control, vol. 55, no. 4, pp. 1024-1028, 2010.

[30] H. R. Feyzmahdavian, T. Charalambous, and M. Johansson, "Exponential stability of homogeneous positive systems of degree one with time-varying delays," IEEE Transactions on Automatic Control, vol. 59, no. 6, pp. 1594-1599, 2014.

[31] Y. Sun, Y. Tian, and X.-J. Xie, "Stabilization of positive switched linear systems and its application in consensus of multiagent systems," IEEE Transactions on Automatic Control, vol. 62, no. 12, pp. 6608-6613, 2017.

[32] Yuangong Sun, Zhaorong Wu, and Fanwei Meng, "Common Weak Linear Copositive Lyapunov Functions for Positive Switched Linear Systems," Complexity, vol. 2018, Article ID 1365960, 7 pages, 2018.

[33] N. Zhang, Y. Sun, and F. Meng, "State bounding for switched homogeneous positive nonlinear systems with exogenous input," Nonlinear Analysis: Hybrid Systems, vol. 29, pp. 363-372, 2018.

[34] H. L. Smith, Monotone dynamical systems: an introduction to the theory of competitive and cooperative systems, ameircan mathematical society, 1995.

[35] V. S. Bokharaie, O. Mason, and F. Wirth, "Stability and positivity of equilibria for subhomogeneous cooperative systems," Nonlinear Analysis. Theory, Methods \& Applications, vol. 74, no. 17, pp. 6416-6426, 2011. 


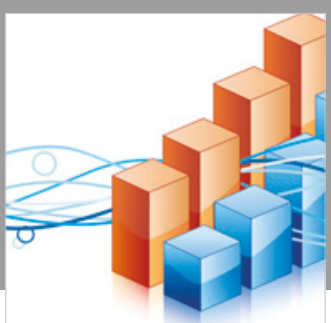

Advances in

Operations Research

\section{-n-m}
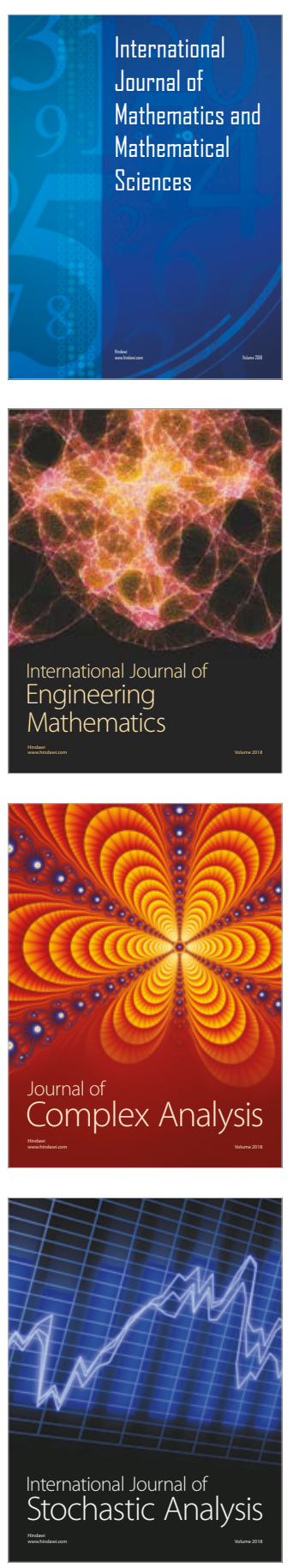
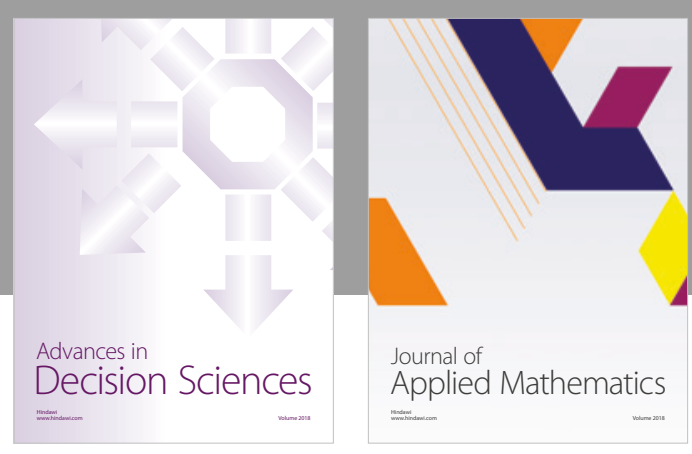

Journal of

Applied Mathematics
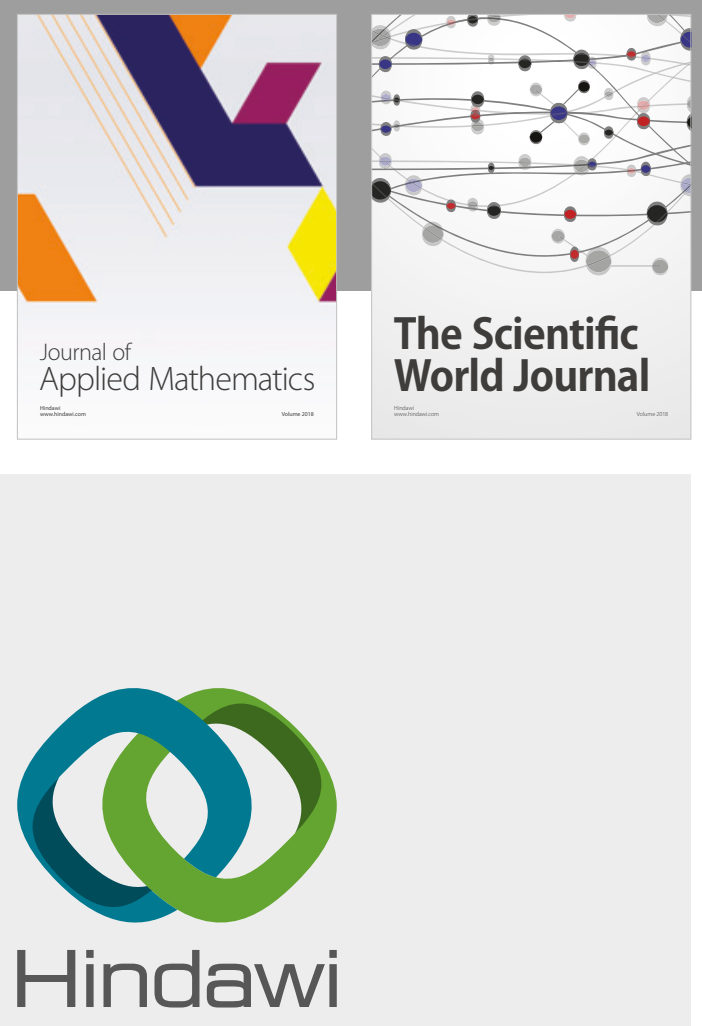

Submit your manuscripts at

www.hindawi.com

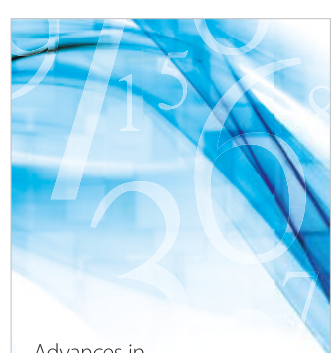

Advances in
Numerical Analysis
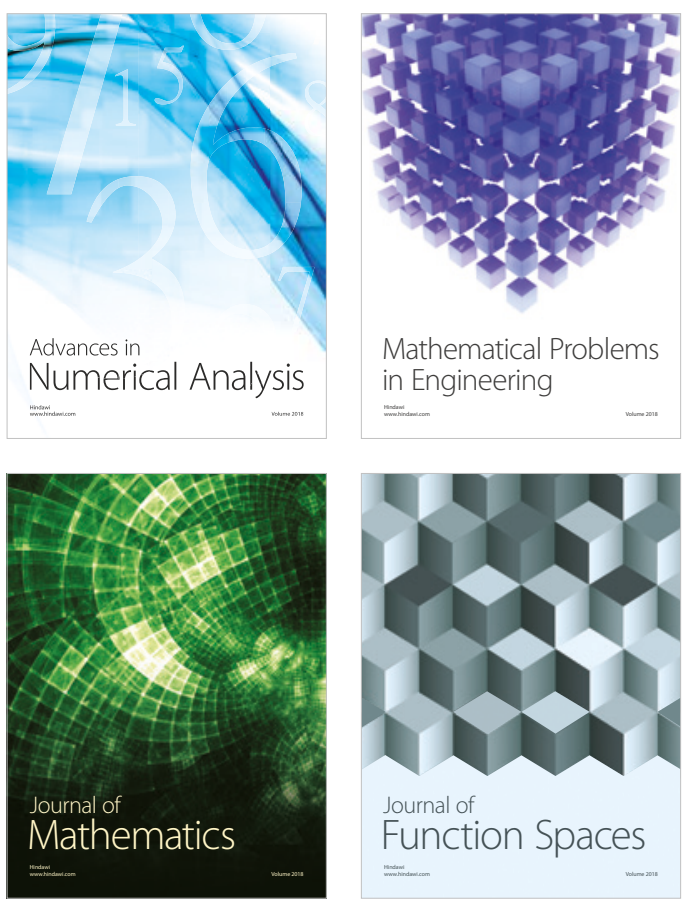

Mathematical Problems in Engineering

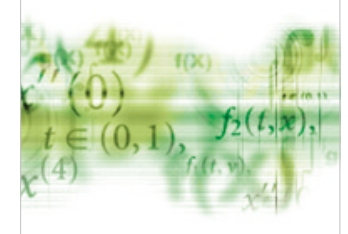

International Journal of

Differential Equations

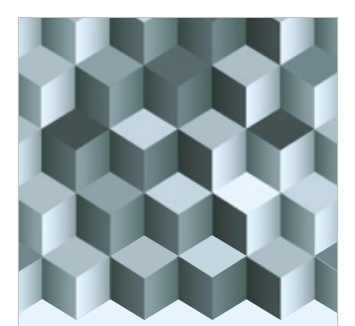

Journal of

Function Spaces
The Scientific

World Journal

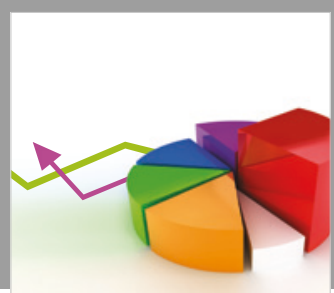

Journal of

Probability and Statistics
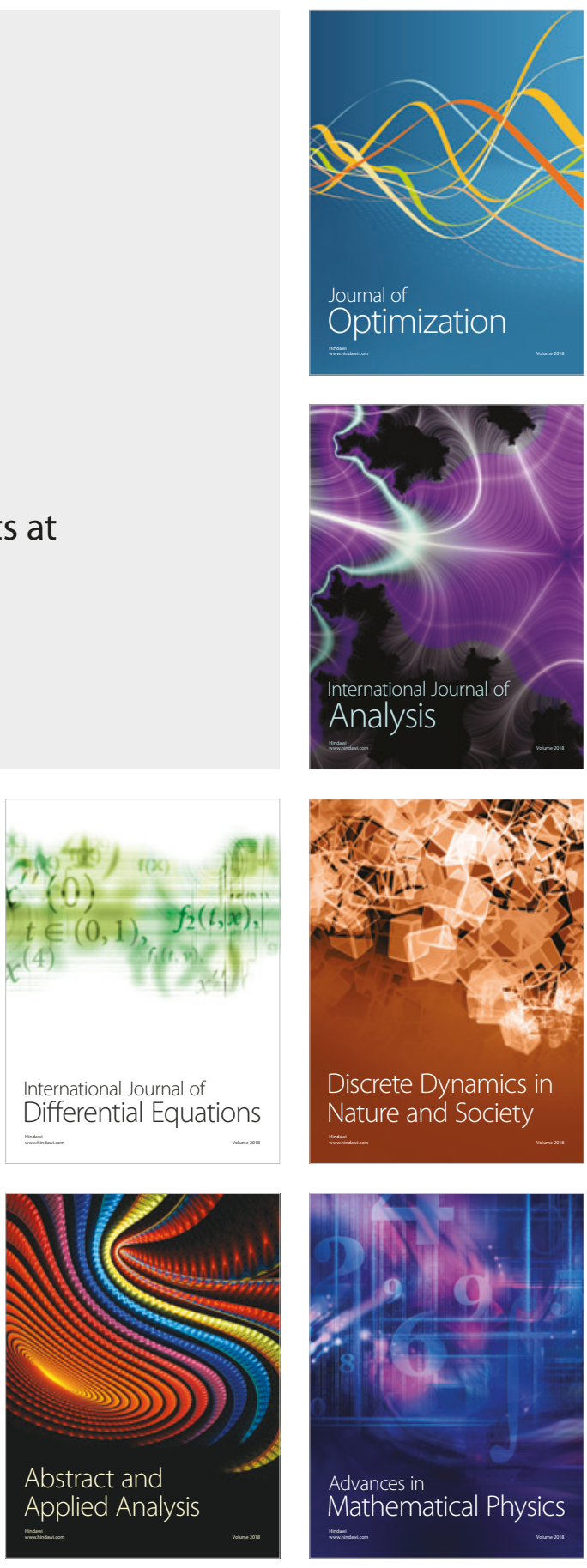